\title{
CRISPR-Cas9-mediated multiplex gene editing in CAR-T cells
}

Cell Research (2017) 27:154-157. doi:10.1038/cr.2016.142; published online 2 December 2016

\section{Dear Editor,}

Chimeric antigen receptor (CAR) $\mathrm{T}$ cell therapy is a promising approach to treat cancer, such as B-cell malignancy [1]. However, the current standard treatment requires autologous adoptive cell transfer, which is expensive and time-consuming. For newborn and elder patients, it is often difficult to obtain enough $\mathrm{T}$ cells with good quality to generate patient-specific CAR-T cells. To make CAR-T therapy more accessible, it is highly desirable to develop an allogeneic adoptive transfer strategy, in which universal CAR-T cells derived from $\mathrm{T}$ cells from healthy donors can be applied to treat multiple patients. For this strategy to work, the $\alpha \beta$ T-cell receptor (TCR) on allogeneic CAR-T cells needs to be eliminated to avoid graft-versus-host-disease (GVHD), and human leukocyte antigens class I (HLA-Is) on CAR-T cells need to be removed to minimize their immunogenicity. Previous studies have shown that mutation in TCR $\alpha$ subunit constant (TRAC) leads to loss of $\alpha \beta$ TCR on T-cell surface [2], and beta-2 microglobulin (B2M) is essential for cell-surface expression of HLA-I heterodimers [3]. Thus, we attempted to target TRAC and $B 2 M$ genes in CAR-T cells. Considering blocking programmed death-1 (PD1) signaling can effectively treat cancers via reversing immunosuppression, we also targeted $P D-1$ in CAR-T cells to render them nonresponsive to PD-1 signaling [4].

To generate universal and more potent CAR-T cells described above, multiple genes need to be eliminated simultaneously. While Torikai et al. $[2,5]$ have used ZFN to knockout TRAC [2] and HLA-A [5] individually in CAR-T cells, they did not test the function of these edited cells in vivo. Although researchers from Cellectis have used TALEN to simultaneously target TRAC and CD52 or deoxycytidine kinase in CD19 CAR-T cells [6, 7] and demonstrated the anti-tumor activity of the edited CAR-T cells in a lymphoma murine model [6], it remains to be tested whether CAR-T cells lacking B2M can still function properly. More importantly, whether clustered regularly interspaced short palindromic repeats (CRISPR) and CRISPR-associated protein 9 (Cas9) system
(CRISPR-Cas9) [8] can be applied to perform multiplex gene editing in CAR-T cells has not been evaluated. Previously, we have demonstrated that up to five genes can be disrupted simultaneously in mouse embryonic stem cells with high efficiency using CRISPR-Cas9 [9]. In this study, we developed protocols to efficiently generate CAR-T cells with two (TRAC and B2M) or three genes (TRAC, B2M, and $P D-1$ ) disrupted and tested their anti-tumor functions in vitro and in vivo.

We designed four sgRNAs targeting the first exon of $T R A C$ and four sgRNAs targeting the first exon of $B 2 M$. For $P D-1$, we designed two sgRNAs and tested one published sgRNA, all targeting the first exon [10]. Cas9 protein $(3 \mu \mathrm{g})$ and in vitro-transcribed sgRNA $(3 \mu \mathrm{g})$ were mixed and then electroporated into human primary CD3 $+\mathrm{T}$ cells isolated from umbilical cord blood. The gene editing efficiency using each sgRNA was quantified by both surveyor assay and tracking of indels by decomposition (TIDE) analysis [11], and the percentage of $\mathrm{T}$ cells losing the expression of each gene was quantified using flow cytometry (Supplementary information, Figure S1A, S1B, and Table S1). The gene-edited cells maintained good proliferation (Supplementary information, Figure S1B iii).

To improve gene editing efficiency, we compared the editing efficiency of using one and two sgRNAs. Consistent with previous published results [12], for targeting $P D-1$ and $B 2 M$, using two most efficient sgRNAs was more effective than using a single sgRNA. However, using the single best sgRNA led to the most efficient TRAC disruption (Supplementary information, Figure S1C). Based on these results, we used five sgRNAs in all the following experiments (two sgRNAs targeting $B 2 M$, two sgRNAs targeting $P D-1$, and one sgRNA targeting TRAC; Figure $1 \mathrm{~A}$ and $1 \mathrm{~B}$ ), and optimized the total amount of reagents used for multiplex gene editing. For generating double-knockout ( $B 2 M$ and TRAC, DKO) and triple-knockout (B2M, TRAC and $P D-1, \mathrm{TKO}) \mathrm{T}$ cells, using $18 \mu \mathrm{g}$ Cas 9 protein along with $18 \mu \mathrm{g}$ sgRNAs resulted in high editing efficiency, while maintaining reasonable cell viability (Supplementary information, 
A
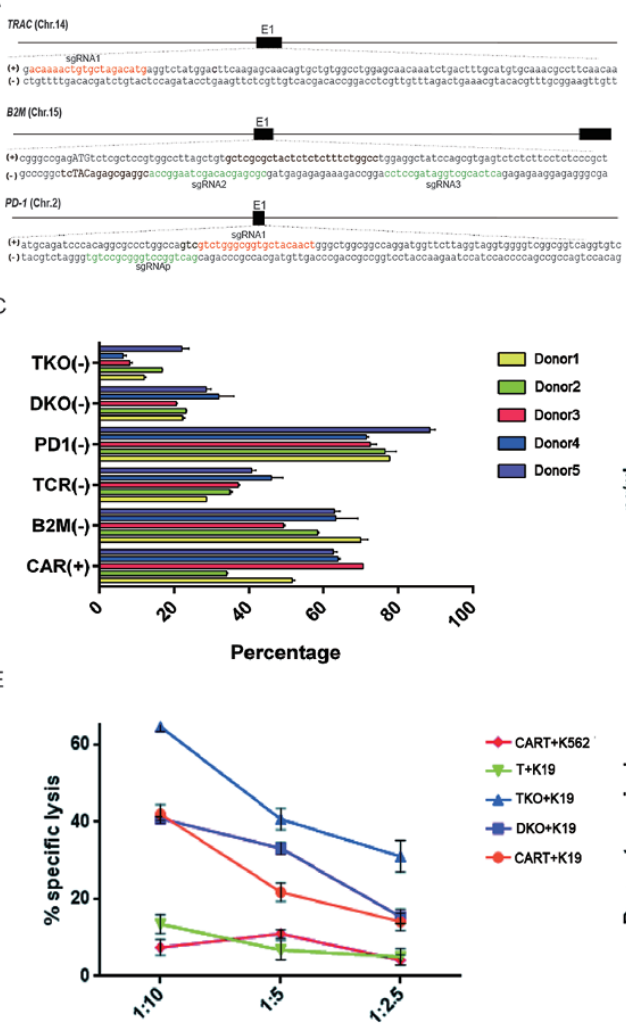

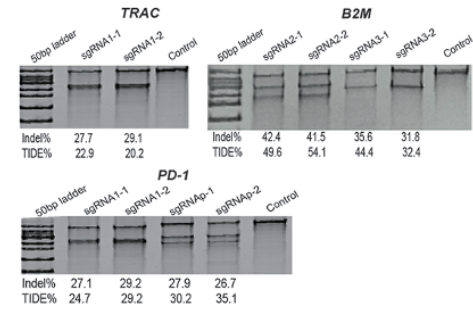

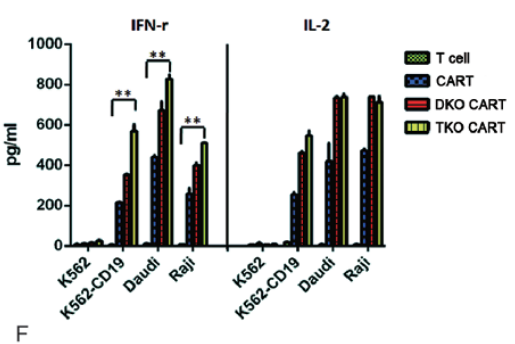

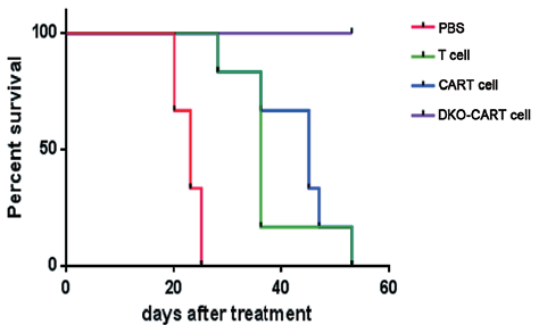

G

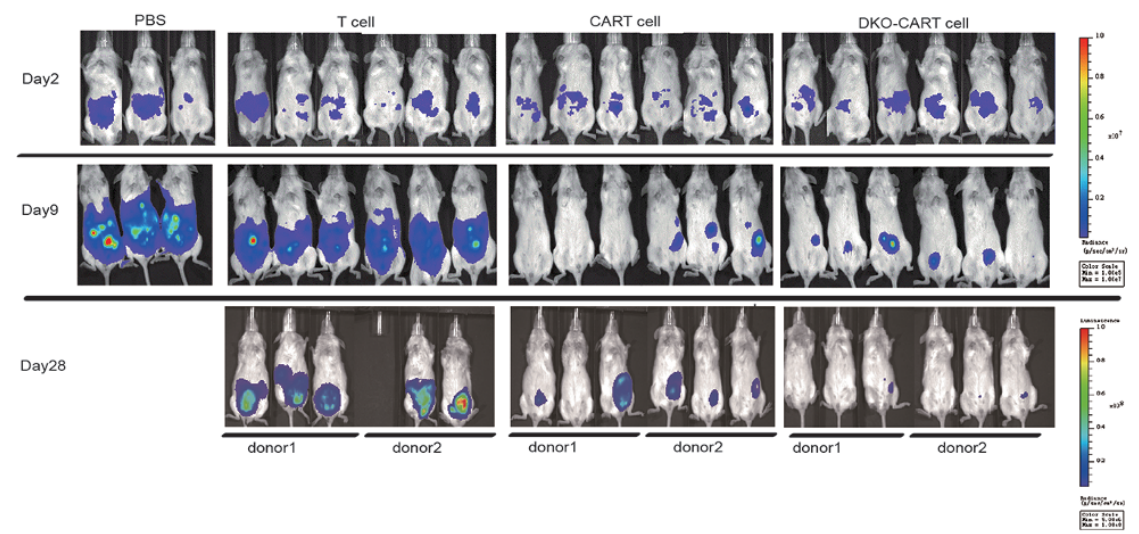

Figure 1 CRISPR-Cas9-mediated multiplex gene editing of TRAC, B2M, and PD-1 in CAR-T cells. (A) Schematic diagram of sgRNA-targeting sites on TRAC, B2M, and $P D$-1. All the targeting sites are within exon1 of each gene. The sgRNA-targeting sites on the sense strand are colored in red whereas those on the antisense strand are colored in green. (B) Quantification of Cas9:sgRNA ribonucleoprotein (Cas9 RNP)-induced indels by surveyor assay and TIDE analysis. Human primary CD3+ T cells $\left(1 \times 10^{6}\right)$ were electroporated with RNP complex ( $3 \mu \mathrm{g}$ Cas 9 protein and $3 \mu \mathrm{g}$ sgRNA) 3 days post stimulation, and collected 7 days post electroporation to quantify mutation frequency. Experiments were performed in two biological replicates. (C) DKO and TKO editing was performed in CAR-T cells from five donors. Flow cytometric analysis of CAR, TRAC, B2M, and PD-1 surface expression in DKO or TKO samples (no enrichment) was performed on day 8 post electroporation (mean $\pm \mathrm{SEM} ; n=2)$. TKO $(-)$ : percentage of triple $($ TRAC, B2M and $P D$-1)-negative cells in TKO samples; DKO (-): percentage of double (TRAC and B2M)-negative cells in DKO samples; PD-1 (-): percentage of $P D$-1-negative cells in TKO samples; TCR (-): percentage of TRAC-negative cells in DKO samples; B2M (-): percentage of B2M-negative cells in DKO samples. CAR (+): CAR-positive cells in in CAR-transduced T cells before gene editing (gene editing did not change the percentage of CAR-positive cells significantly). (D) IL-2 and IFN $\gamma$ production of CAR-T cells from donor 1 (mean $\pm \mathrm{SEM} ; n=2 ;{ }^{* *} P<0.01$ ). Results of two other donors are shown in Supplementary information, Figure S2F i and ii. (E) Flow cytometry-based cytotoxicity assay evaluating the cell lytic activity of T, CAR-T, DKO CAR-T, and TKO CAR-T cells against K562 or K19 cells (mean \pm SEM; $n=2$ ). The $X$-axis indicates the ratio of tumor vs effector (T or CAR-T) cells. (F) Survival rates of xenograft mice receiving T, CAR-T or DKO CAR-T treatment. On day 0 , NOD-Prkdc ${ }^{\text {scid }} \| 2 \mathrm{rg}^{\text {null }}$ (NPG) mice were injected intraperitoneally with 2 $\times 10^{5}$ Raji-ffluc cells. On day 3 , mice received $5 \times 10^{6}$ CAR-T cells, DKO CAR-T cells, T cells, or PBS intraperitoneally. Survival curve of mice 60 days post treatment is shown. (G) Bioluminescent imaging result of NPG mice treated with standard CAR-T and DKO CAR-T cells from two independent donors on days 2,9 , and $28(n=3)$. 
Figure S1D i-iii). To purify the cells losing the expression of the target genes, we labeled the target proteins using PE-conjugated antibodies, and then removed the labeled cells using anti-PE antibody-conjugated beads. By negative selection, we considerably enriched the single-, double-, and triple-negative cells (Supplementary information, Figure S1D iv). We amplified and subcloned each target region from the enriched TKO T cells. Sequencing confirmed that the majority of the clones of $B 2 M(31 / 31,100 \%)$ and TRAC (29/34, 85\%) PCR products were mutants, and all mutations recovered happened precisely at the sgRNA-targeting regions (Supplementary information, Figure S1D v). However, only $64.7 \%$ (22/34) clones of the $P D-1$ PCR products were mutants, indicating that TKO T cells were a mixture of cells with and without $P D-1$ mutations. One possible explanation is that the negative selection-based enrichment method did not work well for enriching T cells with $P D-1$ mutations as $P D-1$ expression was downregulated during $\mathrm{T}$ cell expansion (Supplementary information, Figure S1D iv).

Next, we predicted the top five potential off-target sites for each sgRNA using the Benchling software [13], and genotyped all the 25 sites in the enriched TKO T cells (Supplementary information, Table S1). Using TIDE analysis, we did not detect any mutation at these sites. As mutation in coding region is more likely to be detrimental, we performed exome sequencing analysis of TKO T cells derived from two donors and their corresponding non-edited controls. As expected, the majority of the genetic variations were shared between the edited and control samples from the same donor. The number of unique genetic variations in edited sample is very similar to the control-specific variations, suggesting that most of these variations came from technical error or spontaneous mutations accumulated during cell expansion (Supplementary information, Figure S1E). We scanned $100 \mathrm{bp}$ window of genomic sequences centered at the edited sample-specific genetic variations and could not detect any alignments of sgRNA spacerPAM sequences with $<5 \mathrm{nt}$ mismatches (Supplementary information, Data $\mathrm{S} 1$ ). These results suggest that it is unlikely that these non-target variations were caused by CRISPR-Cas9-mediated gene editing. In addition to off-target indels, chromosomal translocation could happen when multiple DNA double-strand breaks were introduced. We performed quantitative PCR (Supplementary information, Table S1) to detect possible translocation events and did not find any difference between TKO and control samples (data not shown).

We next performed CRISPR-Cas9-mediated gene editing in CAR-T cells. We used anti-CD19 CAR-T cells in following gene editing experiments, as they have been well-characterized and successfully applied for treating CD19+ B-cell leukemia and lymphomas [1]. To optimize the procedure of generating gene-edited CAR-T cells, we tested two different strategies: (1) transduce T cells with anti-CD19 CAR 3 days after $\mathrm{T}$ cell activation, perform CRISPR-Cas9 electroporation on day 5, and harvest cells on day 15 ; (2) perform CRISPR-Cas9 electroporation 3 days after $\mathrm{T}$ cell activation, transduce $\mathrm{T}$ cells on day 5 , and harvest cells on day 15 (Supplementary information, Figure S2A i).

The first strategy (transduction first and electroporation second) generated CAR-T cells with better gene editing efficiency and cell viability (Supplementary information, Figure S2A). Using this protocol, we performed single-, double-, and triple-knockout experiments using CD3+ T cells from five different donors, and obtained relatively similar editing efficiency among these donors (Figure $1 \mathrm{C}$ and Supplementary information, Figure S2B). Gene editing efficiency quantified by surveyor assay and the reduction in gene expression levels determined by flow cytometry in CAR-T cells are similar to those obtained in primary $\mathrm{T}$ cells without CAR transduction (Supplementary information, Figures S1B and S2B). Although gene editing had negative effect on cell proliferation, these edited CAR-T cells can proliferate considerably. 15 days after $\mathrm{T}$ cell activation, standard CAR-T cells expanded around 300-fold, whereas CAR-T cells with multiplex gene editing expanded $\sim 100$-fold (Supplementary information, Figure S2C). After gene editing, the DKO and TKO CAR-T cells were enriched by negative selection using magnetic beads. Although the enrichment is significant, we observed variations among different donors. Enriched DKO cells have a purity of $95 \%$ or higher, whereas enriched TKO cells had triplenegative cells ranging from $57 \%$ to $90 \%$, depending on the donor (Supplementary information, Figure S2D). As certain amount of cells containing wild-type target genes retained after the enrichment, further optimization of the enrichment protocol is necessary for clinical application.

Compared with control CAR-T cells, DKO and TKO CAR-T cells did not show dramatic change in the proportion of CD4 and CD8 cells (Supplementary information, Figure S2E i). To test whether DKO cells have lost TCR function, we measured the proliferative response of these cells after stimulation by cross-linking CD3 with anti-CD3/anti-CD28 beads. Although control CAR-T cells showed robust proliferation, the proliferation of DKO CAR-T cells was dramatically dampened (Supplementary information, Figure S2E ii), indicating loss of TCR function. In addition, the majority of DKO CAR-T cells lost HLA-A expression on the surface (Supplementary information, Figure S2E iii). Taken together, these results 
show that DKO CAR-T cells have lost TCR and HLA-I expression on the cell surface.

Next, we evaluated the cytotoxic function of these gene-edited CAR-T cells in vitro. Similar to standard CAR-T cells, DKO and TKO CAR-T cells released IL-2 and IFN $\gamma$ only when they were co-cultured with CD19-expressing Daudi, Raji, and K562-CD19 (K19) cells. Furthermore, the amount of cytokines released by gene-edited CAR-T cells is similar to or even larger than that produced by standard CAR-T cells (Supplementary information, Figure S2F i and ii). In comparison with standard and DKO CAR-T cells, TKO cells released more IFN $\gamma$ (Figure 1D and Supplementary information, Figure $\mathrm{S} 2 \mathrm{~F}$ i), which is consistent with previously published results on $P D-1$-knockout T cells $[14,15]$. In cell cytotoxicity assays, like unmodified CAR-T cells, DKO and TKO CAR-T cells effectively induced cell death of co-cultured tumor cells in a CAR-dependent manner with TKO CAR-T cells showing higher efficiency (Figure 1E and Supplementary information, Figure $\mathrm{S} 2 \mathrm{~F}$ iii), indicating that CAR-T cells with multiple gene disruption have antitumor activity equivalent or even superior to standard CAR-T cells.

Then we evaluated the antitumor function of the DKO CAR-T cells (TRAC/B2M knockout) using a lymphoma xenograft mouse model. FFLuc-transduced Raji (Raji-ffluc) lymphoma cells were injected intraperitoneally. Mice bearing comparable tumor loads were segregated into groups and then received different treatments intraperitoneally 3 days later. Mice were continually monitored for survival (Figure 1F), and tumor burdens were monitored via luminescence imaging for 1 month (Figure 1G). Tumor size was substantially smaller in all of the mice treated with standard CAR-T cells and DKO CAR-T cells, whereas progressive tumor growth was observed in the control group treated with unmodified $\mathrm{T}$ cells or PBS. These data indicate that CAR-T cells with both TCR and HLA-I eliminated from the cell surface maintained CD19-specific antitumor function, and CRISPR-Cas9-mediated multiplex gene editing did not compromise their potency relative to standard CD19 CAR-T cells. Further research is needed to investigate whether these edited CAR-T cells do not cause GVHD and lose alloantigenicity in humanized immune-competent mouse models.

In conclusion, our study shows that CRISPR-Cas9-mediated multiplex gene editing is readily applicable to CAR-T cells. Although these double (TRAC/B2M)- and triple (TRAC/B2M/PD-1)-negative CAR-T cells need to be further tested for their safety and efficacy in clinical studies, our results suggest that they are promising reagents for cancer therapy.

\section{Acknowledgments}

We would like to thank Mark Wanner (the Jackson Laboratory) for critical review and comments on the manuscript. We would like to thank Junning Wei and Yi Yang (Beijing Cord Blood Bank) for their help in preparing the cord blood samples. This work was supported by the National Natural Science Foundation of China (31471215), the Strategic Priority Research Program of the Chinese Academy of Sciences (XDA01010409), and the State 863 project (2015AA020307). HW is supported by the "Young Thousand Talent Project".

Xiaojuan Liu ${ }^{1, *}$, Yongping Zhang ${ }^{2, *}$, Chen Cheng ${ }^{1,3, *}$, Albert W Cheng ${ }^{4}$, Xingying Zhang ${ }^{1,5}, \mathrm{Na} \mathrm{Li}^{1}$, Changqing Xia ${ }^{2,6}$, Xiaofei $\mathrm{Wei}^{7}$, Xiang Liu ${ }^{1}$, Haoyi Wang ${ }^{1,5,8}$

${ }^{I}$ State Key Laboratory of Stem Cell and Reproductive Biology, Institute of Zoology, The Chinese Academy of Sciences, Beijing, China; ${ }^{2}$ Department of Hematology, Xuanwu Hospital, Capital Medical University, Beijing, China; ${ }^{3}$ Graduate School, University of Science and Technology of China, Hefei, China; ${ }^{4}$ The Jackson Laboratory for Genome Medicine, Farmington, CN, USA; ${ }^{5}$ University of Chinese Academy of Sciences; ${ }^{6}$ Department of Pathology, Immunology and Laboratory Medicine, University of Florida, FL, USA; ${ }^{7}$ Beijing Cord Blood Bank, Beijing, China; ${ }^{8}$ The Jackson Laboratory, Bar Harbor, ME, USA

*These three authors contributed equally to this work.

Correspondence: Haoyi Wang

E-mail: wanghaoyi@ioz.ac.cn

\section{References}

1 Kochenderfer JN, Rosenberg SA. Nat Rev Clin Oncol 2013; 10:267276.

2 Torikai H, Reik A, Liu PQ, et al. Blood 2012; 119:5697-5705.

3 Riolobos L, Hirata RK, Turtle CJ, et al. Mol Ther 2013; 21, 1232 1241.

4 Kamphorst AO, Ahmed R. Curr Opin Immunol 2013; 25:381-388.

5 Torikai H, Reik A, Soldner F, et al. Blood 2013; 122:1341-1349.

6 Poirot L, Philip B, Schiffer-Mannioui C, et al. Cancer Res 2015; 75:3853-3864.

7 Valton J, Guyot V, Marechal A, et al. Mol Ther 2015; 23:1507-1518.

8 Doudna JA, Charpentier E. Science 2014; 346:1258096.

9 Wang H, Yang H, Shivalila CS, et al. Cell 2013; 153:910-918.

10 Schumann K, Lin S, Boyer E, et al. Proc Natl Acad Sci USA 2015: 112:10437-10442.

11 Brinkman EK, Chen T, Amendola M, et al. Nucleic Acids Res 2014; 42, e168.

12 Mandal PK, Ferreira LM, Collins R, et al. Cell Stem Cell 2014; 15:643-652.

13 Hsu PD, Scott DA, Weinstein JA, et al. Nat Biotechnol 2013; 31:827832.

14 Menger L, Sledzinska A, Bergerhoff K, et al. Cancer Res 2016; 76:2087-2093.

15 Su S, Hu B, Shao J, et al. Sci Rep 2016; 6:20070.

(Supplementary information is linked to the online version of the paper on the Cell Research website.) 